\title{
Thinking globally, training locally
}

\author{
Tristan C. Dumbarton, MD • M. Dylan Bould, MBChB
}

Received: 9 February 2016/Revised: 12 February 2016/ Accepted: 11 March 2016/Published online: 30 March 2016

(C) Canadian Anesthesiologists' Society 2016

"It's springtime for surgery!"

Meena Cherian, at the launch of The Lancet

Commission on Global Surgery, April 2015.

Medicine is becoming progressively globalized with increasing movement of healthcare providers and consumers across borders as well as with new technologies and media that facilitate the flow of health information around the world. It is reasonable to postulate that younger and perhaps more technically savvy "digitally native" physicians may have a better understanding of international health inequities than their predecessors, and that this generation may seek global health opportunities in their career paths. In this issue of the Journal, Evans et al. ${ }^{1}$ report on a retrospective survey that asked recently matched anesthesia residents -in the 2014 U.S. residency match - whether international educational opportunities were important in their final choice of residency programs. Global health opportunities were listed as an important factor when choosing a residency program, comparable with factors such as research strength and exposure to simulation-based training. Nevertheless,

Both authors are board members on the not-for-profit Canadian Anesthesiologists' Society International Education Foundation.

Les deux auteurs sont membres du conseil d'administration de la Fondation pour l'éducation internationale de la Société canadienne des anesthésiologistes, un organisme à but non lucratif.

T. C. Dumbarton, MD

Department of Anesthesiology, Perioperative Medicine and Pain Management, Dalhousie University, Halifax, NS, Canada

M. D. Bould, MBChB ( $\square)$

Department of Pediatric Anesthesia, Children's Hospital of Eastern Ontario, 401 Smyth Rd, Ottawa, ON K1H 8L1, Canada e-mail: dbould@cheo.on.ca aspiring residents viewed global health opportunities as less important than the overall reputation of the program, geographic location, and general impressions from the interview. Comparison with these other factors is arguably uninformative, particularly as they are either non-specific or not easily modifiable. Regardless, the authors argue that residency programs should consider adding international education opportunities to curricula, as this generation of residents seem to indicate more interest in global health than ever before. Indeed, 38\% of respondents already had exposure to an international educational experience prior to residency, and $48 \%$ expressed a desire for global health as a part of their future career.

Interestingly, the U.S. National Residency Matching Program (NRMP) conducted a similar survey in the same year. $^{2}$ The methodology of the NRMP survey is not explained in detail, but the response rate was slightly higher than that in the Evans et al. ${ }^{1}$ report, with more detailed questions asked about program selection. Some of these results conflict with the survey findings of Evans et al. When NRMP survey respondents included opportunity for international experience as a factor, they ranked it highly; however, the survey suggests that few residents actually cited this factor as "influential" in selecting a program. Despite some conflicting findings, it seems reasonable to conclude that opportunities for involvement in global health during residency are important to a significant proportion of trainees. Along with education and clinical excellence, research is one of the three traditional pillars of academic medicine. Should it be surprising then if residents see global health opportunities as comparable to research opportunities when selecting residency programs?

For many years, anesthesia and surgery have received scant attention within the global health community, while infectious disease and maternal/child health have seen 
prioritization. Pervasive myths about global perioperative care exist; many of these have recently been addressed and debunked (below). Appropriately, surgery and anesthesia are now receiving increasing international attention and resources. They are the new "hot topics" in global health.

\section{Myth 1: Surgical care and anesthesia are not priorities for low- and middle-income countries}

The surgical burden of disease can be difficult to quantify it's easier to measure the number of people with (human immunodeficiency virus) HIV than the number of people who can be effectively treated by surgery. Nevertheless, the burden of surgical conditions is huge and accounts for almost one-third of the global burden of all disease. ${ }^{3}$ Trauma is the largest component of disease amenable to surgical intervention and results in more deaths each year than HIV, malaria, and tuberculosis combined. ${ }^{4}$ Comprehensive obstetric care also requires the capacity for surgical intervention. This means that maternal and newborn health, a key focus in global health, will never reach its full potential without surgical capacity. Surgical needs in low- and middle-income countries (LMICs) also include treatment for congenital malformations and a large burden of disease due to cancer. The recognition of perioperative care as a priority for LMICs led to the adoption of resolution WHA 68.15 —"Strengthening emergency and essential surgical care and anesthesia as a component of universal health coverage" - at the $68^{\text {th }}$ World Health Assembly in May 2015. ${ }^{5}$ This resolution is an important acknowledgement by governments across the world that surgery and anesthesia strengthen health systems and are necessary parts of universal health coverage.

\section{Myth 2: Surgical care and anesthesia are too expensive for low- and middle-income countries}

Perioperative care requires infrastructure, well-maintained equipment, consumables, and the support of numerous healthcare professions and medical specialties. It is natural to assume that anesthesia and surgical care are unlikely to be cost-effective in the poorest countries in the world and that these countries should focus instead on basics such as clean water, immunizations, or infectious diseases such as malaria and HIV. Nevertheless, surgical care has been found to be highly cost-effective in LMICs. Adult male circumcision for the prevention of HIV has costeffectiveness comparable to interventions such as vaccines for polio and bed nets impregnated with insecticide for the prevention of transmission of malaria. ${ }^{6}$ Cost-effectiveness is often described in terms of dollars per disability-adjusted life year (DALY) or the cost per year of life saved from early death or disability. A Cesarean delivery for obstructed labour is around $\$ 300$ per DALY, which is often less expensive than antiretroviral therapy for $\mathrm{HIV}^{6}$ or oral rehydration therapy. ${ }^{7}$

Achieving the goal set out by the Lancet Commission on Global Surgery -i.e., $80 \%$ of the world's population will have access to safe surgical care by 2030 - will require an estimated investment of US\$350 billion. ${ }^{3}$ Nevertheless, comparable amounts have previously been invested in other areas, such as HIV medicine, with great success. The potential return on investment is orders of magnitude higher. It's estimated that the lost gross domestic product due to surgical disease over the same period of time would be over $\$ 12$ trillion. ${ }^{5}$ It is too expensive for LMICs not to invest in safe perioperative care.

\section{Myth 3: It is not feasible to build surgical capacity in low- and middle-income countries}

The scale of the problem is vast -5 billion people lack access to safe, timely, and affordable surgical care. ${ }^{5}$ Training the additional 2.2 million anesthesiologists, obstetricians, and surgeons required to provide such surgical care in LMICs may be seen by some as an insurmountable obstacle. Some may feel that "brain drain" of trained specialists away from their countries of origin is inevitable and will nullify the effect of an investment in healthcare professional education in LMICs. Nevertheless, there is an established model of partnerships between institutions in high-income countries and LMICs to build capacity for perioperative care. From 1985-2005, the Canadian Anesthesiologists' Society International Education Foundation (CASIEF) worked in partnership with the Tribhuvan University Teaching Hospital in Kathmandu. In 1985, there were only seven anesthesiologists in Nepal for a population of 16 million. Over the following 20 years, 62 anesthesiologists were trained, with the majority choosing to remain in Nepal. Currently, the anesthesia community in that country is thriving. ${ }^{8}$ Although non-physician anesthesia providers will likely be required to provide the majority of anesthesia care in LMICs for many years to come, this kind of program creates leadership in anesthesia. It allows LMICs to develop their own solutions to safe perioperative care, including the coordination and training of nonphysician anesthetists.

Similar successful partnerships exist in Rwanda between CASIEF, the American Society of Anesthesiologists' Global Humanitarian Outreach, and the University of Rwanda- ${ }^{9}$ and in Zambia - between the UK's Tropical Health and Education Trust and the 
University of Zambia. ${ }^{10}$ Recruitment and retention efforts by a partnership of Global Partners in Anesthesia and Surgery and Makerere University increased the number of anesthesia trainees from two in 2007 to 19 in 2011, all of whom graduated and remained in Uganda. ${ }^{11}$ These programs should serve as "proof-of-concept" for this model of training for a global anesthesia workforce.

\section{Myth 4: A focus on global health has little benefit for the anesthesia community in high-income countries}

Aside from philanthropic interests, the analysis from the Lancet Commission arguably creates an ethical imperative for scaling up global health programs in anesthesia. Nevertheless, many anesthesiologists in high-income countries presume that all gains from global health partnerships go to the lower-resource partner. In fact, there are also benefits to the high-income partner. In his seminal report on healthcare partnerships, Nigel Crisp wrote that these partnerships build strong relationships that stand us all "in good stead in a changing and risky world", and partners from high-income countries can learn about managing their own healthcare needs. ${ }^{12}$ "Reverse innovation", describing the flow of ideas from emerging to developed economies, is well established in the business community and is gaining increasing traction in healthcare. ${ }^{13}$ There are opportunities for collaborative academic and research pursuits. Collaboration brings unparalleled educational experiences for both trainees and staff, particularly in the intrinsic CanMEDs roles, such as health advocacy, collaboration, and leadership ${ }^{14}$ which can be underemphasized in training at home. Returning trainees are more likely to engage in work with underprivileged populations at home. ${ }^{15}$

So, if Evan $e t$ al.'s findings really do represent emerging future leadership in global anesthesia, it couldn't come at a better time. These future leaders and partners in global health need support from North American universities and training institutions in partnership with other organizations with experience in capacity building in perioperative care (e.g., CASIEF, the Canadian Network for International Surgery, and the Society for Obstetricians and Gynaecologists of Canada). It is notable that many residents in the survey by Evans et al. ${ }^{1}$ were more interested in providing clinical service internationally than in involvement in teaching or research, but this focus is unlikely to meet the challenges set out by the Lancet Commission. Instead, we encourage development of leadership for programs focusing on building truly independent capacity for safe anesthesia care in LMICs. To achieve this, Canadian universities should support their faculty in developing and contributing to these programs, recognizing that these activities are aligned with broader and more inclusive theories of scholarship. ${ }^{16}$ Healthcare partnerships should be conducted in a scholarly way, as we know that the problems of global anesthesia can't be fixed without data. ${ }^{17}$ Institutions from high-income countries should leverage their well-developed skill sets in education, research, data management, and patient safety to improve care in LMICs. Anesthesia has a daunting challenge ahead of it on the global stage, but it also has a huge opportunity to make a difference - perhaps we are where our colleagues in HIV medicine were more than 20 years ago. History may not judge us kindly if we fail to meet this challenge.

\section{Penser mondialement, former localement}

«C'est le printemps de la chirurgie! »

Meena Cherian, à l'occasion du lancement de The Lancet Commission on Global Surgery (Commission du journal The Lancet sur la chirurgie internationale) en avril 2015.

La médecine s'internationalise progressivement avec un mouvement croissant des professionnels de la santé et des bénéficiaires au-delà des frontières, ainsi qu'avec les nouvelles technologies et médias qui facilitent la circulation de l'information de santé à travers le monde. Il est raisonnable de postuler que les médecins plus jeunes et peut-être plus orientés vers la technologie, «numériquement natifs », ont sans doute une meilleure connaissance que leurs prédécesseurs des inégalités en matière de soins dans le monde et que cette génération est susceptible de rechercher des possibilités de carrières internationales dans le domaine de la santé. Dans ce numéro du Journal, Evans et coll. ${ }^{1}$ rapportent une enquête rétrospective au cours de laquelle les auteurs ont demandé à des résidents en anesthésie récemment appariés - dans le cadre des placements de résidents aux États-Unis en 2014 - si les opportunités d'éducation internationale étaient importantes dans leur choix définitif de programmes de résidence. Les opportunités internationales dans le domaine de la santé ont été décrites comme un facteur important dans le choix du programme de résidence, à égalité avec d'autres facteurs tels que la solidité de la recherche et l'exposition à une formation stimulante. Néanmoins, les aspirants résidents estimaient que les opportunités internationales étaient moins importantes que la réputation globale du programme, sa localisation géographique et les impressions générales tirées de l'entretien. La comparaison avec ces autres facteurs ne nous apporte pas beaucoup d'informations dans la mesure où ils ne sont pas spécifiques ou facilement modifiables. 
Toutefois, les auteurs discutent le fait que les programmes de résidence devraient envisager d'ajouter des possibilités d'éducation internationale à leur curriculum car cette génération de résidents semble montrer plus d'intérêt que jamais dans la santé mondiale. De fait, $38 \%$ des répondants ont déjà été exposés à une expérience éducative internationale avant la résidence et $48 \%$ ont exprimé un désir de santé à l'international dans le cadre de leur carrière future.

Il est intéressant de noter que le NRMP (U.S. National Residency Matching Program) à mené aux États-Unis une enquête similaire la même année. ${ }^{2}$ La méthodologie de l'enquête du NRMP n'est pas expliquée en détail, mais le taux de réponse a été légèrement supérieur à celui du rapport d'Evans et coll. ${ }^{1}$ avec des questions plus détaillées sur le choix des programmes. Certains résultats ne concordent pas avec les constatations de l'enquête d'Evans et coll. Quand les répondants à l'enquête du NRMP ont inclus la possibilité d'une expérience internationale, ils ont classé ce facteur assez haut dans leur liste; cependant, l'enquête suggère que peu de résidents ont véritablement indiqué que ce facteur avait de l'influence sur la sélection d'un programme. En dépit de certaines constatations contradictoires, il semble raisonnable de conclure que les possibilités d'implication dans la santé internationale au cours de la résidence sont importantes pour un pourcentage significatif de stagiaires. À côté de l'éducation et de l'excellence clinique, la recherche est l'un des trois piliers traditionnels de la médecine universitaire. Doit-on alors s'étonner si les résidents considèrent les opportunités internationales dans le domaine de la santé de la même manière que les possibilités de recherche quand ils choisissent des programmes de résidence?

Pendant de nombreuses années, l'anesthésie et la chirurgie ont reçu peu d'attention de la part de la communauté médicale internationale, alors que les maladies infectieuses et la santé mère - enfants étaient devenues prioritaires. Il existe des mythes persistants concernant les soins périopératoires dans le monde; nombre d'entre eux ont été récemment abordés et déboulonnés (ci-dessous). Heureusement, la chirurgie et l'anesthésie reçoivent maintenant une attention et des ressources internationales croissantes. $\mathrm{Ce}$ sont les nouveaux thèmes en vogue en matière de santé globale.

\section{Mythe $n^{\circ} 1$ : Les soins chirurgicaux et anesthésiques ne sont pas une priorité pour les pays à revenus faibles et moyens}

Le fardeau chirurgical de la maladie peut être difficile à quantifier; il est plus facile de compter le nombre de personnes atteintes par le VIH (virus de l'immunodéficience humaine) que le nombre de personnes pouvant être efficacement traitées par la chirurgie. Néanmoins, le poids des affections chirurgicales est considérable et représente pratiquement le tiers du fardeau global englobant toutes les maladies. ${ }^{3}$ Les traumatismes représentent le plus grand pourcentage d'affections pouvant être traitées chirurgicalement et ils entraînent chaque année plus de décès que le $\mathrm{VIH}$, le paludisme, et la tuberculose réunis. ${ }^{4}$ Des soins obstétricaux complets nécessitent également une capacité d'intervention chirurgicale. Cela signifie que la santé de la mère et du nouveau-né, un objectif essentiel de la santé globale, n'atteindra jamais son plein potentiel sans une capacité chirurgicale. Les besoins chirurgicaux dans les pays à revenus faibles et moyens (PRFM) incluent également le traitement des malformations congénitales et la lourde morbidité des cancers. La reconnaissance des soins périopératoires en tant que priorité pour les PRFM a conduit à l'adoption de la résolution WHA 68.15 sur le " développement des soins chirurgicaux d'urgence, les soins chirurgicaux essentiels et l'anesthésie en tant que composantes de la couverture sanitaire universelle », à la $68^{e}$ Assemblée mondiale de la santé en mai $2015 .^{5}$ Cette résolution est une reconnaissance importante par les gouvernements du monde entier que la chirurgie et l'anesthésie renforcent les systèmes de santé et sont des éléments nécessaires de la couverture sanitaire universelle.

\section{Mythe $n^{\circ} 2$ : Les soins chirurgicaux et anesthésiques sont trop coûteux pour les pays à revenus faibles et moyens}

Les soins périopératoires nécessitent une infrastructure, un équipement bien entretenu, des consommables et le soutien de nombreuses professions de la santé et de spécialités médicales. Il est naturel de supposer que les soins anesthésiques et chirurgicaux ne sont pas susceptibles d'être rentables dans les pays du monde les plus pauvres et que ces pays devraient plutôt se concentrer sur des éléments de base, tels que l'eau propre, les vaccinations ou les maladies infectieuses comme le paludisme et le VIH. Néanmoins, on a pu montrer que les soins chirurgicaux sont extrêmement rentables dans les PRFM. La circoncision des hommes adultes pour la prévention du VIH a un rapport coût-efficacité comparable à des interventions telles que la vaccination contre la polio et les moustiquaires imprégnées d'insecticides pour la prévention de la transmission du paludisme. ${ }^{6}$ Le rapport coût-efficacité est souvent décrit en termes de dollars par année de vie ajustée pour l'invalidité (DALY disability-adjusted life year) ou le coût par année de vie sauvée d'une mort prématurée ou d'une invalidité. Un 
accouchement par césarienne face à un travail qui ne peut aboutir coûte environ 300 \$ par DALY, ce qui est souvent moins coûteux qu'un traitement antirétroviral contre le $\mathrm{VIH}^{6}$ ou une réhydratation par voie orale. ${ }^{7}$

Pour atteindre le but fixé par la commission de The Lancet sur la chirurgie globale (c.-à-d., faire en sorte que $80 \%$ de la population mondiale aient accès à des soins chirurgicaux sécuritaires d'ici 2030) nécessitera un investissement estimé de 350 milliards de dollars (US). ${ }^{3}$ Néanmoins, des sommes comparables ont déjà été investies avec un grand succès dans d'autres domaines, tel que les médicaments contre le VIH. Le retour sur investissement possible est d'un ordre de grandeur très supérieur. On estime que le produit intérieur brut perdu à cause d'affections chirurgicales pendant la même période dépasserait 12 milliards de dollars. ${ }^{5}$ Ce montant est trop élevé pour que les PRFM n'investissent pas dans des soins périopératoires sécuritaires.

\section{Mythe $n^{\circ} 3$ : Bâtir une capacité chirurgicale dans les pays à revenu faible et moyen n'est pas faisable}

Le problème est de grande ampleur: cinq milliards de personnes n'ont pas accès à des soins chirurgicaux sécuritaires, d'un coût abordable et dans des délais normaux. ${ }^{5}$ La formation de 2,2 millions d'anesthésiologistes, obstétriciens et chirurgiens supplémentaires nécessaires pour fournir de tels soins chirurgicaux dans les PRFM peut paraitre un obstacle insurmontable. Certains peuvent estimer que «l'exode de cerveaux » depuis leur pays d'origine est inévitable et que cela annulera l'effet d'un investissement dans la formation de professionnels en soins de santé dans les PRFM. Il existe pourtant un modèle de partenariat entre les institutions de pays à revenus élevés et les PRFM pour ce qui concerne la création d'une capacité de soins périopératoires. De 1985 à 2005, la Fondation d'éducation internationale de la Société canadienne des anesthésiologistes (FÉI SCA) a travaillé en partenariat avec l'hôpital universitaire Tribhuvan à Katmandou. Il n'y avait en 1985 que sept anesthésiologistes au Népal pour une population de 16 millions d'habitants. Au cours des 20 années suivantes, 62 anesthésiologistes ont été formés et la majorité d'entre eux a choisi de rester au Népal. La communauté anesthésique est actuellement florissante dans ce pays. ${ }^{8}$ Bien que des fournisseurs de soins d'anesthésie non-médecins seront probablement requis pour assurer la plupart des soins anesthésiques dans les PRFM pendant encore de nombreuses années, ce type de programme créé un leadership en anesthésie. Cela permet aux PRFM de développer leurs propres solutions pour des soins périopératoires sécuritaires, y compris la coordination et la formation d'anesthésistes non-médecins.
Un partenariat similaire réussi existe au Rwanda — entre la FÉI SCA, l'initiative humanitaire globale (Global Humanitarian Outreach) de la Société américaine des anesthésiologistes et l'université du Rwanda -9 et en Zambie - entre le trust sur l'éducation et de la santé tropicale (Tropical Health and Education Trust) du Royaume-Uni et l'université de Zambie. ${ }^{10}$ Les efforts de recrutement et de fidélisation par un partenariat d'intervenants internationaux en anesthésie et chirurgie, et l'université Makerere ont fait passer le nombre de stagiaires en anesthésie de deux, en 2007, à 19 en 2011, lesquels ont tous été diplômés en Ouganda et continuent à y vivre. ${ }^{11}$ Ces programmes doivent servir de " preuve de concept » pour ce modèle de formation du personnel d'anesthésie dans le monde.

\section{Mythe $n^{\circ} 4$ : Se focaliser sur la santé globale n'a que peu d'effet pour la communauté de l'anesthésie dans les pays à hauts revenus}

À côté des aspects philanthropiques, l'analyse de la Commission de The Lancet crée un impératif éthique discutable de renforcement des programmes de santé globale en anesthésie. Néanmoins, de nombreux anesthésiologistes des pays à revenus élevés présument que tous les bénéfices des partenariats de santé globale iront au partenaire le moins fortuné. En fait, le partenaire ayant un revenu élevé peut aussi en tirer des bénéfices. Dans son rapport novateur sur les partenariats dans le domaine des soins de santé, Nigel Crisp a écrit que ces partenariats créent des relations solides qui nous mettent en « très bonne posture dans un monde changeant et dangereux » et que les partenaires de pays à revenus élevés peuvent apprendre comment gérer leurs propres besoins sanitaires. $^{12}$ "Inverser le sens de l'innovation », décrivant le flux des idées depuis les économies émergentes vers les économies développées est une notion bien établie dans le monde des affaires et qui gagne de plus en plus de force dans le monde de la santé. ${ }^{13}$ Il existe des possibilités de collaboration universitaire et de projets de recherche. La collaboration apporte des expériences éducatives sans équivalent, autant pour les stagiaires que pour le personnel, notamment dans les fonctions CanMED intrinsèques, telles que la défense de la santé, la collaboration et le leadership ${ }^{14}$ sur lesquels la formation dans le pays d'origine peut ne pas insister suffisamment. Les stagiaires rentrant chez eux sont plus susceptibles de travailler auprès de populations défavorisées dans leur pays. ${ }^{15}$

Donc, si les constatations d'Evan et coll. représentaient véritablement un futur leadership émergent de l'anesthésie dans le monde, elles n'arriveraient pas à un meilleur moment. Ces futurs chefs de file et partenaires de la 
santé globale ont besoin du soutien des universités nord-américaines et des institutions de formation en partenariat avec d'autres organisations ayant l'expérience requise pour développer des soins périopératoires (par exemple, l'ÉIF SCA, le Réseau canadien pour la chirurgie internationale et la Société des obstétriciens et gynécologues du Canada). Le fait que de nombreux résidents ayant répondu à l'enquête d'Evans et coll. ${ }^{1}$ aient été plus intéressés à fournir des services cliniques à l'étranger qu'à s'impliquer dans l'enseignement ou la recherche est quelque chose de notable, mais ce centre d'intérêt a peu de chances de parvenir à relever les défis identifiés par la commission de The Lancet. En revanche, nous encourageons le développement du leadership pour des programmes se focalisant sur la création d'une capacité vraiment indépendante de soins anesthésiques sécuritaires dans les PRFM. Pour y parvenir, les universités canadiennes devraient soutenir leur personnel enseignant dans l'élaboration de ces programmes et y contribuer, reconnaissant que ces activités correspondent à des théories plus larges et inclusives de l'enseignement. ${ }^{16}$ Les partenariats dans le domaine de la santé doivent être menés d'une manière réfléchie, car nous savons que les problèmes de l'anesthésie globale ne peuvent pas être résolus sans données. ${ }^{17}$ Les établissements de pays à revenus élevés devraient user de leurs ensembles de compétences abouties dans les domaines de l'éducation, de la recherche, de la gestion et des données et de la sécurité des patients pour améliorer les soins dans les PRFM. L'anesthésie est maintenant confrontée à un défi gigantesque sur la scène internationale, mais elle a également l'unique occasion de faire une différence - nous nous trouvons peut-être là où nos collègues spécialistes du VIH se trouvaient il y a 20 ans. Le jugement de l'Histoire pourrait ne pas être tendre avec nous si nous ne parvenons pas à relever ce défi.

Acknowledgement Thanks to Dr. Patty Livingston for her feedback on an earlier version of this manuscript.

Funding The authors received no funding for the preparation of this manuscript.

Conflicts of interest None declared.

Editorial responsibility This submission was handled by Dr. Hilary P. Grocott, Editor-in-Chief, Canadian Journal of Anesthesia.

Financement Les auteurs n'ont reçu aucun financement pour la préparation de ce manuscrit.

Conflits d'intérêts aucun déclaré.

Responsabilité éditoriale Cette soumission a été gérée par le Dr Hilary P. Grocott, Rédacteur en chef du Journal canadien d'anesthésie.

\section{References}

1. Evans FM, Mallepally NR, Dubowitz G, et al. Factors influencing anesthesia residency selection: impact of global health opportunities. Can J Anesth 2016; 63: DOI:10.1007/s12630016-0629-7.

2. National Resident Matching Program. National Resident Matching Progam, Results and Data: 2013 Main Residency Match ${ }^{\circledR}$. National Resident Matching Program, Washington, DC, 2013. Available from URL: http://www.nrmp.org/match-data/ nrmp-historical-reports (accessed February 2016).

3. Meara JG, Leather AJ, Hagander L, et al. Global Surgery 2030: evidence and solutions for achieving health, welfare, and economic development. Lancet 2015; 386: 569-624.

4. World Health Organization. Injuries and Violence - the Facts - 2010. Available from URL: http://apps.who.int/iris/bitstream/10665/ 44288/1/9789241599375_eng.pdf (accessed February 2016).

5. Price $R$, Makasa E, Hollands $M$. World Health Assembly Resolution WHA68.15: "Strengthening Emergency and Essential Surgical Care and Anesthesia as a Component of Universal Health Coverage" - Addressing the public health gaps arising from lack of safe, affordable and accessible surgical and anesthetic services. World J Surg 2015; 39: 2115-25.

6. Chao TE, Sharma K, Mandigo M, et al. Cost-effectiveness of surgery and its policy implications for global health: a systematic review and analysis. Lancet Glob Health 2014; 2: e334-45.

7. Grimes CE, Henry JA, Maraka J, Mkandawire NC, Cotton M. Cost-effectiveness of surgery in low- and middle-income countries: a systematic review. World J Surg 2014; 38: 252-63.

8. Shrestha BM, Rana NB. Training and development of anesthesia in Nepal - 1985 to 2005. Can J Anesth 2006; 53: 339-43.

9. Twagirumugabe T, Carli F. Rwandan anesthesia residency program: a model of north-south educational partnership. Int Anesthesiol Clin 2010; 48: 71-8.

10. Kinnear JA, Bould MD, Ismailova F, Measures E. A new partnership for anesthesia training in Zambia: reflections on the first year. Can J Anesth 2013; 60: 484-91.

11. Lipnick $M$, Mijumbi $C$, Dubowitz $G$, et al. Surgery and anesthesia capacity-building in resource-poor settings: description of an ongoing academic partnership in Uganda. World J Surg 2013; 37 : 488-97.

12. Crisp N. Global health partnerships, the UK contribution to health in developing countries. 2007. Available from URL: http:// webarchive.nationalarchives.gov.uk/20080814090248/dh.gov.uk/ en/Publicationsandstatistics/Publications/PublicationsPolicyAnd Guidance/DH_065374 (accessed February 2016).

13. Depasse JW, Lee PT. A model for 'reverse innovation' in health care. Global Health 2013; 9: 40.

14. Frank JR, Snell L, Sherbino J (editors). CanMEDS 2015 Physician Competency Framework. Ottawa: Royal College of Physicians and Surgeons of Canada; 2015. Available from URL: http://www. royalcollege.ca/portal/page/portal/rc/common/documents/canmeds/ framework/canmeds_full_framework_e.pdf (acccessed February 2016).

15. Liaw W, Bazemore A, Xierali I, Walden J, Diller P. Impact of global health experiences during residency on graduate practice location: a multisite cohort study. J Grad Med Educ 2014; 6: 451-6.

16. Boyer EL. Scholarship Reconsidered: Priorities of the Professoriate. Lawrenceville, NJ: Princeton University Press; 1990 .

17. Weiser TG, Makasa EM, Gelb AW. Improving perioperative outcomes in low-resource countries: it can't be fixed without data. Can J Anesth 2015; 12: 1239-43. 